\title{
A Rare Case of Left Main Coronary Artery Atresia
}

\author{
Anthony H. Kashou ${ }^{a}$, Nabil Braiteh ${ }^{\mathrm{a}}$, Hisham E. Kashou ${ }^{\mathrm{a}, \mathrm{b}}$
}

\begin{abstract}
Left main coronary artery (LMCA) atresia remains one of the most rare congenital coronary anomalies. On average, there is less than one reported case each year. Because patients with LMCA atresia that make it to adulthood tend to remain asymptomatic and often are unaware of their condition, the diagnosis is often made incidentally. We report a case of LMCA atresia in a 76-year-old male with a history of seizure disorder and medication non-compliance who presented to the hospital with acute coronary syndrome in the setting of status epilepticus. Workup revealed ST elevation and minimally elevated troponin levels. Hours later, troponin levels continued to trend up, but the ST elevation resolved. Catheterization and coronary angiography revealed a dominant right coronary artery and absent LMCA. Aortography confirmed the diagnosis of LMCA atresia.
\end{abstract}

Keywords: Left main coronary artery atresia; Angiography; Congenital coronary anomaly; Acute coronary syndrome

\section{Introduction}

While congenital heart defects are the most common cause of all birth defects, congenital coronary artery disease is quite rare. In fact, left main coronary artery (LMCA) atresia remains one of the least frequently observed variations. The overall incidence is estimated between $0.6 \%$ and $1.3 \%$ [1-3]. A recent review found a total of only 53 cases of LMCA atresia ever reported in the literature, including both pediatric and adult cases [3]. The authors found more than half of the patients survived into adulthood with a presentation of either angina or atypical chest pain.

Although the LMCA is atretic, the coronary arteries tend to retain a normal anatomic arrangement around the heart, often without evidence of atherosclerotic disease [4]. Because the coronary ostium is absent and the left main trunk ends blindly, the right coronary artery (RCA) becomes the dominant vessel of blood flow to the entire heart, relying on right-to-left col-

Manuscript submitted October 21, 2017, accepted November 3, 2017

aDepartment of Cardiology, United Health Services Hospitals, Wilson Regional Medical Center, Johnson City, NY, USA

${ }^{\mathrm{b}}$ Corresponding Author: Hisham E. Kashou, Department of Cardiology, United Health Services Hospitals, Wilson Regional Medical Center, 30 Harrison St \#250, Johnson City, NY 13790, USA. Email: hishamekashou@gmail.com

doi: https://doi.org/10.14740/jmc2946w lateral circulation to perfuse the left side of the heart. A poor prognosis in adults can be expected with myocardial bridging, excessive coronary angulation, as well as a limited number of and/or narrow caliber collateral vessels. Herein, we report an extremely rare case of LMCA atresia in a 76-year-old male with acute coronary syndrome in the setting of status epilepticus.

\section{Case Report}

A 76-year-old Caucasian male with seizure disorder and coronary artery disease risk factors of age, diabetes, hypertension, dyslipidemia, and tobacco abuse presented to the hospital unresponsive following generalized tonic-clonic movements. His wife found him shaking on the floor for $30 \mathrm{~min}$. She does not recall any complaints of chest pain or shortness or breath, but notes he is non-compliant with his medications.

The patient was intubated. Physical exam was significant for tachycardia (122 beats/min). Electrocardiogram (ECG) showed sinus tachycardia and mild ST elevation in leads I, $\mathrm{aVL}$, and $\mathrm{V}_{3}-\mathrm{V}_{6}$ with reciprocal ST depression in leads II, III, and aVF. Troponin T level was $0.038 \mathrm{ng} / \mathrm{mL}$. Seizure medication serum levels were undetectable. Computed tomography of the brain showed no intracranial pathology. The patient was transferred to the intensive care unit and managed medically. Repeat troponin levels showed an increase to $7.4 \mathrm{ng} / \mathrm{mL}$ after the first $6 \mathrm{~h}$ and then again to $16.4 \mathrm{ng} / \mathrm{mL}$ over the next $6 \mathrm{~h}$. Bedside echocardiogram (ECHO) was normal. Repeat ECG showed resolution of the initial acute changes.

After the patient's condition stabilized, left heart catheterization and coronary angiography were performed. A large dominant RCA with luminal irregularities and atretic LMCA were found (Fig. 1). There was a predominant right-to-left blood flow over the heart with retrograde flow on the left side. The distal RCA was supplying right-to-left collaterals to the left circumflex and left anterior descending (LAD) arteries. The proximal segment of the LAD artery showed $80-90 \%$ stenosis. These findings were confirmed by aortography (Fig. 2).

On the basis of these findings, the patient was diagnosed with LMCA atresia. The patient was asymptomatic and managed medically on discharge. At his 6-month and 1-year follow-up appointments, he remained medically compliant and asymptomatic with normal left ventricular systolic function.

\section{Discussion}

Due to the patient's initial workup and absence of cardiac symptoms, it is likely that the seizure secondary to medication 

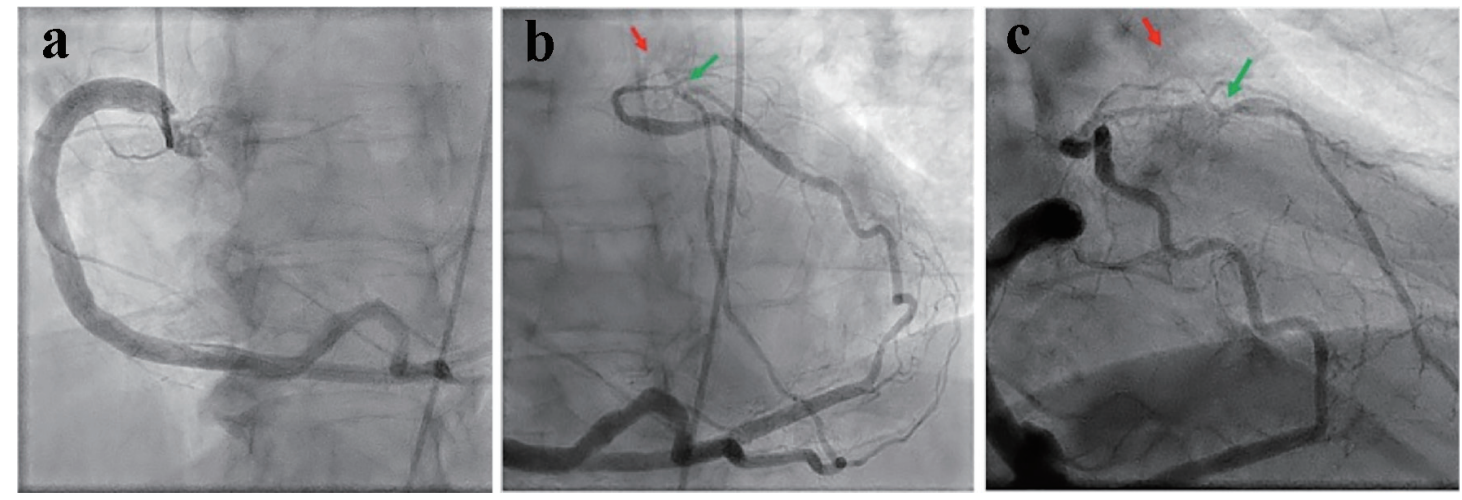

Figure 1. Coronary angiography showing a dominant RCA with mild luminal irregularities and no significant disease (a). Distal RCA supplying right-to-left collaterals to the left circumflex and LAD arteries with an atretic LMCA (red arrows) and an $80-90 \%$ stenosed proximal LAD artery segment (green arrows (b, c)).

non-compliance precipitated his presentation and incidental finding of LMCA atresia. We propose two hypotheses for the presence of acute coronary syndrome: coronary artery vasospasm and/or occlusion.

Any stressful event (e.g., seizure) can trigger coronary artery vasospasm. In patients with LMCA atresia, the RCA is dominant artery supplying the heart; hence, even a small reduction of blood flow to the heart from coronary artery vasospasm can cause myocardial injury and elevated troponin levels. The transient ECG findings are also characteristic of coronary artery vasospasm, which resolved once the stressor was removed.

The other possibility is coronary artery occlusion secondary to rupture of preexisting atherosclerotic plaque. While the majority of patients with LMCA atresia often lack atherosclerotic disease, it is not improbable for this 76-year-old male with cardiac risk factors to have atherosclerotic disease. The

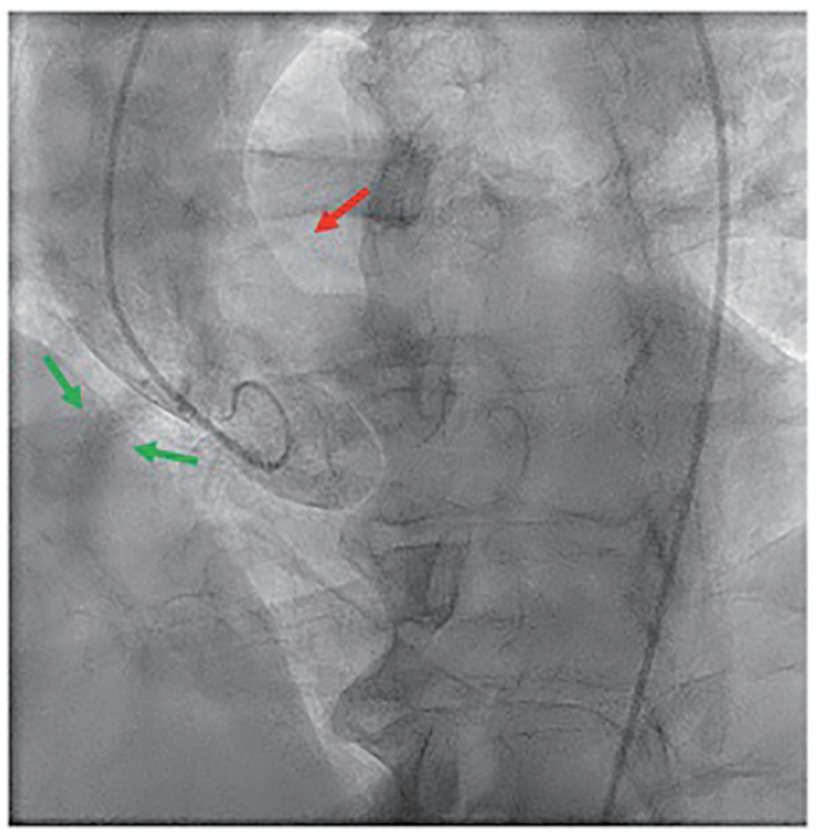

Figure 2. Aortography revealing the presence of the RCA (green arrows) and absence of the LMCA (red arrow). stress induced by the seizure may have precipitated rupture of a preexisting plaque and occluded the already $80-90 \%$ stenosed LAD artery. The initial ECG findings and progressive rise in troponin levels from myocardial injury support this. Although repeat ECG showing resolution of the acute changes seems to suggest otherwise.

Due to the infrequent incidence of LMCA atresia, optimal management of such patients remains relatively unclear. In adults, stressful events appear to trigger coronary artery vasospasm and/or occlusion that result in its presentation and incidental finding with acute coronary syndrome. In this case, medication compliance appeared to prevent recurrence. Management strategies should be focused on controlling any precipitating factors and medically treating any underlying conditions.

\section{Conflict of Interest}

Authors have no conflict of interest.

\section{Funding Support}

The work was not supported or funded by any drug company.

\section{References}

1. Angelini P, Velasco JA, Flamm S. Coronary anomalies: incidence, pathophysiology, and clinical relevance. Circulation. 2002;105(20):2449-2454.

2. Yildiz A, Okcun B, Peker T, Arslan C, Olcay A, Bulent Vatan M. Prevalence of coronary artery anomalies in 12,457 adult patients who underwent coronary angiography. Clin Cardiol. 2010;33(12):E60-64.

3. Tanawuttiwat T, O'Neill BP, Schob AH, Alfonso CE. Left main coronary atresia. J Card Surg. 2013;28(1):37-46.

4. Musiani A, Cernigliaro C, Sansa M, Maselli D, De Gasperis C. Left main coronary artery atresia: literature review and therapeutical considerations. Eur J Cardiothorac Surg. 1997;11(3):505-514. 